\title{
Detecting Gravitational Waves from Quasi-Continuous Sources: the German-British Project GEO 600
}

\author{
Albrecht Rüdiger for the GEO team \\ Max-Planck-Institut für Quantenoptik, Garching/Hannover, Germany
}

\begin{abstract}
At five sites, laser-interferometric detectors, with armlengths ranging from 0.3 to $4 \mathrm{~km}$, are being built. By using advanced optical technologies early on, the German-British project GEO 600, although only intermediate in size $(600 \mathrm{~m})$, has good chances for a competitive sensitivity. Particularly the use of the so-called signal recycling technique will allow a search for faint sources of only slowly varying frequency (pulsars, close binaries). First science runs of GEO 600 are expected in the year 2001.
\end{abstract}

\section{The GEO 600 Concept}

The particular topology of the GEO 600 interferometer is characterized by the use of a four-pass optical delay line and signal recycling (see Fig. 1). The beam of a (highly stabilized) laser is divided into two orthogonal beams that would encounter different phase modulation by a gravitational wave, to be detected after recombination at the beam splitter. The mirror $M_{P R}$ enhances the light power circulating in the interferometer, the mirror $M_{S R}$ enhances the signal.
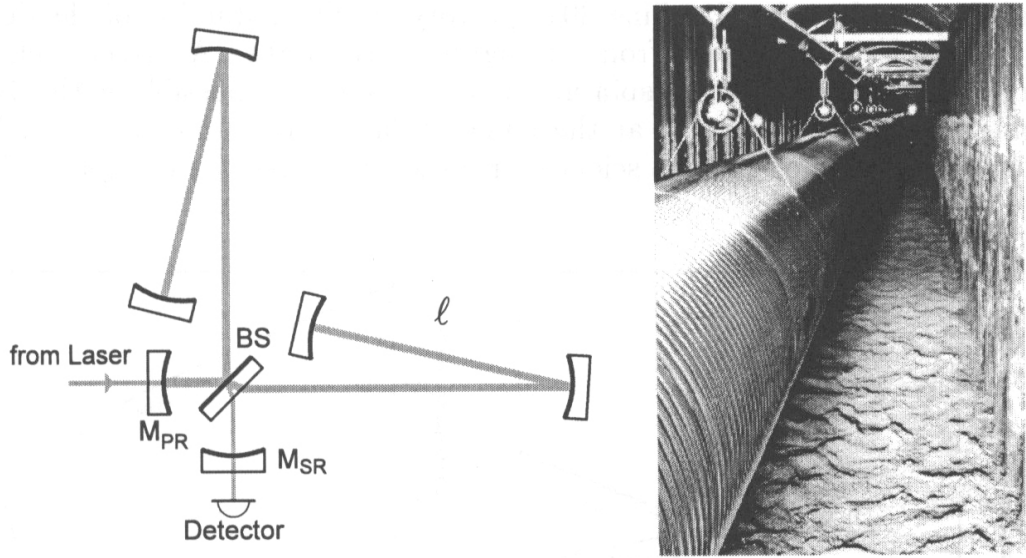

Figure 1. Left: The optical configuration of the GEO600 interferometer, with two extra mirrors $M_{P R}$ and $M_{S R}$ for power and signal recycling. Right: One of the interferometer arms, a $600 \mathrm{~m}$ vacuum tube made of thin corrugated steel, suspended in an accessible trench. 


\section{Seeing Gravitational Waves from Pulsars}

Rapidly rotating cosmic bodies (neutron stars, pulsars) emit gravitational radiation, provided they are not symmetric to their axis of rotation. Pulsars would be typical examples of such 'skewed rotation'. These quasi-periodic gravitational waves would be observable if they are in the frequency band of the detectors, typically between 10 and $3000 \mathrm{~Hz}$.

At five sites, large-scale gravitational wave detectors using laser interferometry have reached or are nearing completion: LIGO at two sites in the US with interferometers of $4 \mathrm{~km}$ each, the French-Italian collaboration VIRGO with a $3 \mathrm{~km}$ detector near Pisa, and on a somewhat smaller scale, with $600 \mathrm{~m}$ and $300 \mathrm{~m}$ armlengths: the German-British antenna GEO 600 near Hannover, and the Japanese antenna TAMA 300 near Tokyo.

For GEO 600, the smaller armlength was not a matter of choice, but one of necessity. The site (owned by Hannover University) cannot accommodate a larger antenna, and the funds did not allow buying or leasing ground elsewhere.

Among the interferometric detectors currently being built, it is particularly GEO 600 that lends itself to the detection of quasi-periodic waves. This is so because GEO 600 is the only detector that can be tuned to a given pulsar frequency. Construction (civil engineering) of GEO600 is finished, the vacuum housing (with two tubes $600 \mathrm{~m}$ in length) and the clean-room conditions have been successfully tested, and first optical components are being installed.

\section{Signal Recycling is the Trick}

GEO 600 exploits a number of advanced techniques that will be used in the other detectors only much later. The scheme of signal recycling allows a narrowbanded enhancement of the sensitivity. Signal recycling has recently been successfully tested in the Garching $30 \mathrm{~m}$ prototype. The reduction of the dominating shot noise can be seen from the figure to the right. A further refinement (for tracking the frequency spin-up of binary stars) is possible with 'dynamic tuning'. Extensive research at the GEO 600 laboratories has also significantly reduced thermal noise. First scientific runs are expected for the year 2001.
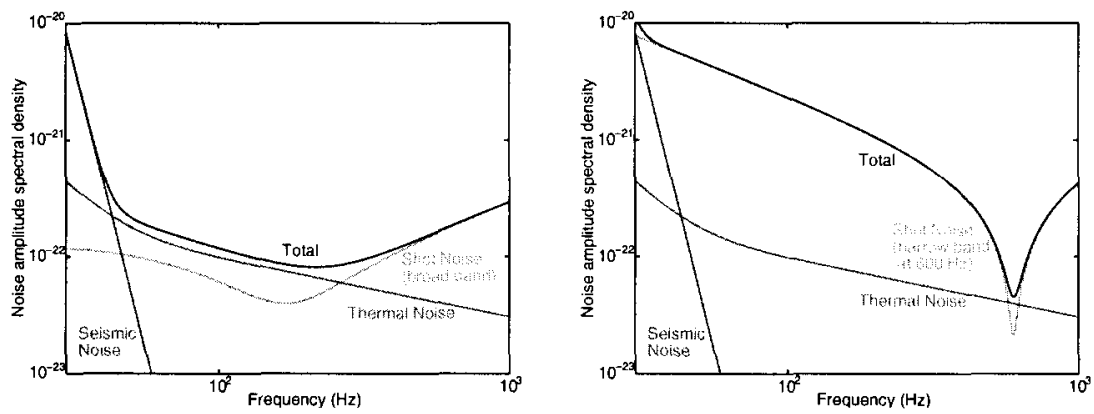

Figure 2. Noise spectral density of GEO 600, shown for two operating modes: for broadband (left) and narrowband operation (right). 